\title{
A Case of Cerebral Salt Wasting Syndrome in Neuromyelitis Optica Spectrum Disorder
}

\author{
Young Min Paek, Jae Jung Lee, Pamela Song, Hee Kyung Park, Joong-Yang Cho \\ Department of Neurology, Inje University Ilsan Paik Hospital, Inje University College of Medicine, Goyang, Korea
}

\begin{abstract}
Neuromyelitis optica spectrum disorder (NMOSD) may present with area postrema syndrome, which is characterized by intractable vomiting and hiccups. Hyponatremia is common in NMOSD and is mostly associated with the syndrome of inappropriate antidiuretic hormone secretion (SIADH). In contrast to SIADH, cerebral salt wasting syndrome (CSWS) causes hyponatremia, which is associated with severe natriuresis and extracellular volume depletion in patients with cerebral disease. To our knowledge, hyponatremia associated with CSWS has not been reported in a patient with NMOSD. Here, we describe a NMOSD presenting with hyponatremia, which may be caused by CSWS following area postrema syndrome.
\end{abstract}

Keywords: Cerebral salt wasting syndrome; Neuromyelitis optica; Intractable vomiting; Hyponatremia

\section{INTRODUCTION}

Neuromyelitis optica spectrum disorder (NMOSD) is an inflammatory, demyelinating syndrome in the central nervous system (CNS) and includes optic neuritis, acute myelitis, area postrema syndrome, and acute brainstem syndrome [1]. NMO-immunoglobulin $\mathrm{G}(\mathrm{IgG})$ is reported to be a biomarker of NMOSD and binds selectively to the water channel protein, aquaporin 4 (AQP4). Neurological symptoms usually correlate with the as specific cerebral region. Involvement of dorsal medulla (an AQP4rich region) may present with area postrema syndrome, which is characterized by intractable vomiting and hiccups [2]. Therefore, intractable vomiting and hiccups with AQP4-seropositivity in a patient strongly support the diagnosis of NMOSD [1]. For any neurological disorders, hyponatremia is the most common form of electrolyte imbalance in patients with neurologic disorders, including NMOSD [3]. The syndrome of inappropriate antidiuretic hormone secretion (SIADH) is most common cause of hyponatremia, which secretion of inappropriate antidiuretic hormone impairs water excretion despite changes in plasma volume is the cause. However, cerebral salt wasting syndrome (CSWS) causes hyponatremia associated with renal loss of sodium and volume depletion in patients with CNS diseases [2]. An accurate diagnosis of hyponatremia is necessary because the different treatments are required. When NMOSD presents with hyponatremia following severe vomiting, etiology may be challenging, but early identification of etiology may be crucial for proper treatment.

To our knowledge, CSWS in a patient with NMOSD has not been reported. We describe a NMOSD case, with uncontrolled hyponatremia due to CSWS.

\section{CASE REPORT}

A 22-year-old man was admitted due to intractable vomiting and hiccups lasting for 4 weeks. He was previously healthy besides a history of recurrent oral ulcers. Despite symptomatic treatments under the diagnosis of acute gastroenteritis, intractable vomiting and hiccups persisted. Eventually, headache and fever developed. The brain computed tomography was normal while the cerebrospinal fluid (CSF) analysis revealed 30 white blood cells/ $\mu \mathrm{L}$ (refer-
Correspondence to: Joong-Yang Cho

Department of Neurology, Inje University Ilsan Paik Hospital, Inje University College of Medicine, 170 Juhwa-ro, Ilsanseo-gu, Goyang 10380, Korea

Tel: +82-31-910-7929, Fax: +82-31-910-7368, E-mail: joongyangcho@gmail.com

Co-correspondence to: Hee Kyung Park

Department of Neurology, Inje University Ilsan Paik Hospital, Inje University College of Medicine, 170 Juhwa-ro, Ilsanseo-gu,

Goyang 10380, Korea

Tel: +82-31-910-7943, Fax: +82-31-910-7368, E-mail: jenhkpark@gmail.com

Received: Jun. 27, 2017 / Accepted after revision: Nov. 8, 2017
(C) 2017 Soonchunhyang Medical Research Institute This is an Open Access article distributed under the terms of the Creative Commons Attribution Non-Commercial License (http://creativecommons.org/licenses/by-nc/4.0/) 
ence range, $0-5$ white blood cells $/ \mu \mathrm{L}$ ) with $95 \%$ of lymphocyte, normal total protein level (17 mg/dL), and normal glucose level (59 $\mathrm{mg} / \mathrm{dL}$ ). This results suggested viral meningitis and he was treated conservatively.

He was transferred to Inje University Ilsan Paik Hospital for further evaluation and management. Upon arrival, he complained of severe thirst, and physical examination revealed to have a dehydrated tongue and axillary skin with decreased skin turgor. Laboratory tests disclosed the following: white blood cells 3,920/ $\mu \mathrm{L}$, hemoglobin $15.6 \mathrm{~g} / \mathrm{dL}$, hematocrit $42.7 \%$, blood urea nitrogen $9 \mathrm{mg} /$ $\mathrm{dL}$, creatinine $0.69 \mathrm{mg} / \mathrm{dL}$, sodium $\left(\mathrm{Na}^{+}\right) 121 \mathrm{mEq} / \mathrm{L}$ (reference range, $136-145 \mathrm{mEq} / \mathrm{L}$ ), potassium $\left(\mathrm{K}^{+}\right) 3.9 \mathrm{mEq} / \mathrm{L}$ (reference range, $3.5-5.1 \mathrm{mEq} / \mathrm{L}$ ), chloride $\left(\mathrm{Cl}^{-}\right) 88 \mathrm{mEq} / \mathrm{L}$ (reference range, 98-104 mEq/L), and serum osmolarity $251 \mathrm{mOsm} / \mathrm{kg}$ (reference range, $270-295 \mathrm{mOsm} / \mathrm{kg}$ ). Tests for his spot urine electrolytes and osmolality were as follows: $\mathrm{Na}^{+} 211 \mathrm{mEq} / \mathrm{L}, \mathrm{K}^{+} 62.7 \mathrm{mEq} / \mathrm{L}, \mathrm{Cl}^{-}$ $122 \mathrm{mEq} / \mathrm{L}$, and osmolarity $920 \mathrm{mOsm} / \mathrm{kg}$. He was initially started on water restriction with no optimal changes in serum sodium. As he lost about $3 \mathrm{~kg}$ of his body weight reaching $70 \mathrm{~kg}$ from $73 \mathrm{~kg}$, he also experienced progressively increasing urine output. His volume depletion was accompanied by urinary sodium loss, which led to serum hyponatremia. Intractable vomiting persisted and brain magnetic resonance imaging (MRI) was performed to detect underlying other cause.

Brain MRI showed high signal intensities involving dorsal medulla, hypothalamus, and third ventricle area on T2-weighted and fluid attenuated inversion recovery images (Fig. 1). A week later, the patient complained of paresthesia in his right arm and cervical
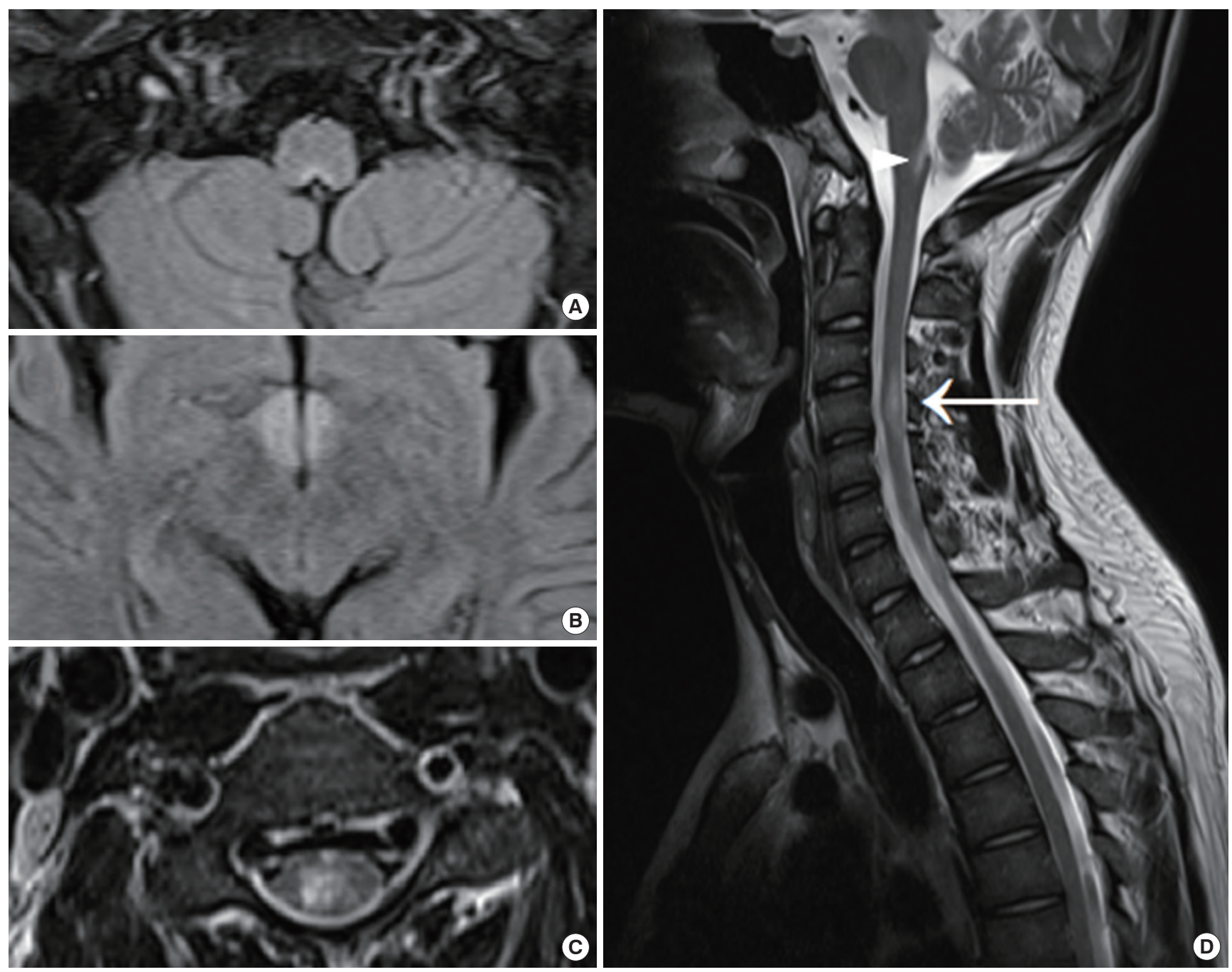

Fig. 1. Brain MRI showed hyperintense lesions in the dorsal medulla (A) and the hypothalamus (B) on fluid attenuated inversion recovery images. MRI of the cervical cord displayed high signal intensities from the third through fifth cervical segments (arrow) and in the medulla (arrow head) (C, D). MRI, magnetic resonance imaging. 
MRI disclosed hyperintense and swollen lesions from the third through fifth cervical segments on a T2-weighted image (Fig. 1).

Brain MRI features were suggestive of NMO and serological anti-AQP4 antibody was resulted in positive. In addition, the antibody to double-stranded DNA, anti-nuclear antibody with a speckled pattern, slightly decreased the level of serum complement factor C3 (80 mg/dL), and leukopenia $(3,290 / \mu \mathrm{L})$ were found. The patient was finally diagnosed as NMOSD with CSWS. After 5 days of intravenous methylprednisolon ( $1 \mathrm{~g} / \mathrm{kg} /$ day), these symptoms improved.

\section{DISCUSSION}

This case has important clinical implications in terms of the diagnostic challenges and the treatment of severe hyponatremia. In this patient, for the first two weeks, acute gastroenteritis was the first diagnosis. Later on, viral meningitis was the second diagnosis after CSF analysis along with a headache and fever. Based on distinct lesions in the dorsal medulla and spinal cord accompanied with AQP4-seropositivity, a final diagnosis of NMOSD with AQP4-IgG was made [1]. In previous reports [2,3], intractable hiccups and vomiting are initial presentations in about $16 \%-43 \%$ of NMOSD patients. Thus, in revised NMOSD criteria, area postrema syndrome is included in core clinical manifestation [1]. Nevertheless, it is a diagnostic challenge for the physician to diagnose a previously healthy young man complaining of gastrointestinal symptoms only as NMOSD in the very early stage.

It is crucial to identify the cause of hyponatremia since the treatment strategies may differ according to the etiologies. At first, the intractable vomiting was considered to be the primary cause of hyponatremia. SIADH seemed to be the cause of hyponatremia [4]. Additionally, brain MRI displayed a lesion in the hypothalamus, which is demonstrated in SIADH. However, he was not responsive to water restriction, the main treatment of SIADH. Moreover, physical signs and laboratory findings were suggestive of hy- povolemic state. This patient had decreased skin turgor, thirst, weight loss, and low blood pressure and increased urine output and sodium. Accordingly, CSWS might be the most plausible cause of hyponatremia. SIADH is associated with a euvolemic or slightly hypervolemic state, whereas CSWS is more likely to be associated with a hypovolemic state [5]. In CSWS, urine output and sodium markedly increase but, in SIADH, the excretion of sodium and water are offset by water retention [5]. The management of CSWS begins with the treatment of the underlying neurologic cause. This patient also made a remarkable recovery of hyponatremia after administration of methylprednisolone. The goal of the treatment in CSWS is to restore decreased serum sodium levels and intravascular volume caused by natriuresis and diuresis [5]. CSWS requires vigorous salt replacement in order to compensate for renal salt wasting using either isotonic or hypertonic saline [5].

In conclusion, this case highlights the diagnostic challenge for physicians when presented with a young man with gastrointestinal symptoms which could be caused by NMOSD. In addition, CSWS should be considered as the possible cause of severe hyponatremia in NMOSD.

\section{REFERENCES}

1. Wingerchuk DM, Banwell B, Bennett JL, Cabre P, Carroll W, Chitnis T, et al. International consensus diagnostic criteria for neuromyelitis optica spectrum disorders. Neurology 2015;85:177-89.

2. Apiwattanakul M, Popescu BF, Matiello M, Weinshenker BG, Lucchinetti $\mathrm{CF}$, Lennon VA, et al. Intractable vomiting as the initial presentation of neuromyelitis optica. Ann Neurol 2010;68:757-61.

3. Yachoui R, Kolasinski SL, Han BK. Intractable vomiting as an initial presentation of lupus-related neuromyelitis optica. J Clin Rheumatol 2013; 19:154-5.

4. Pu S, Long Y, Yang N, He Y, Shan F, Fan Y, et al. Syndrome of inappropriate antidiuretic hormone secretion in patients with aquaporin-4 antibody. J Neurol 2015;262:101-7.

5. Cerda-Esteve M, Cuadrado-Godia E, Chillaron JJ, Pont-Sunyer C, Cucurella $\mathrm{G}$, Fernandez M, et al. Cerebral salt wasting syndrome: review. Eur J Intern Med 2008;19:249-54. 